\title{
Period changes of the long-period cataclysmic binary EX Draconis
}

\author{
L. Pilarčík ${ }^{1}$, M. Wolf ${ }^{1}$, P. A. Dubovský ${ }^{2}$, K. Hornoch ${ }^{3}$, and L. Kotková ${ }^{3}$ \\ 1 Astronomical Institute, Faculty of Mathematics and Physics, Charles University Prague, 18000 Praha 8, V Holešovičkách 2, \\ Czech Republic \\ e-mail: wolf@cesnet.cz \\ 2 Vihorlat Observatory, Mierova 4, 06601 Humenné, Slovakia \\ 3 Astronomical Institute, Academy of Sciences, Fričova 298, 25165 Ondřejov, Czech Republic
}

Received 30 August 2011/ Accepted 22 January 2012

\section{ABSTRACT}

\begin{abstract}
The cataclysmic variable star EX Dra is a relatively faint but frequently investigated eclipsing dwarf nova. In total 35 new eclipses were measured photometrically as part of our long-term monitoring of interesting eclipsing systems. Using published and new mid-eclipse times obtained between 2004 and 2011 we constructed the observed-minus-calculated diagram. The current data present 21 years of period modulation with a semi-amplitude of $2.5 \mathrm{~min}$. The eclipse timings show significant deviations from the best sinusoidal fit, which indicates that this ephemeris is not a complete description of the data. The fractional period change is roughly $\Delta P / P=3 \times 10^{-6}$.
\end{abstract}

Key words. binaries: close - binaries: eclipsing - stars: dwarf novae - stars: individual: EX Draconis - novae, cataclysmic variables

\section{Introduction}

Cataclysmic variables $(\mathrm{CVs})$ are interacting binary systems where the late-type star (the secondary) overfills its Roche lobe and transfers matter to a white dwarf (the primary) via an accretion disc. If the donor star has a lower mass than the white dwarf, the mass transfer will lead to an increase in the orbital separation and size of the Roche lobe and the mass transfer will stop. The CVs must lose their angular momentum to sustain the mass transfer process. There are two possible mechanisms of losing angular momentum: gravitational radiation (for $P_{\text {orb }}<2$ h) and magnetic braking via the secondary star's wind (for $P_{\text {orb }}>3 \mathrm{~h}$ ). This process of decreasing orbital period can be seen in observed-minus-calculated $(\mathrm{O}-\mathrm{C})$ eclipse timings. Almost all CVs (with well-sampled O-C covering more than a decade) shows cyclical changes of the orbital period (the period decrease could be hidden by cyclical change). The most promising explanation of this cyclical change seems to be existence of a solar-type magnetic activity cycle in the secondary star (e.g. Applegate 1992; Lanza et al. 1998).

The dwarf nova EX Draconis is a frequently studied eclipsing binary system with an orbital period of about $5 \mathrm{~h}$ and deep eclipses (1.5 mag). EX Dra was detected in the Hamburger Quasar Survey (Bade et al. 1989) and was shown to be an eclipsing dwarf nova by Barwig et al. (1993). The spectroscopic observations were published by Fiedler et al. (1997). They found that spectral lines of both components are visible in emission as well as in absorption. From radial velocities they derived precise masses of both components $M_{1}=(0.75 \pm 0.02) M_{\odot}$ and, $M_{2}=(0.56 \pm 0.02) M_{\odot}$. The first eclipse study was presented by Baptista et al. (2000), who revised the linear ephemeris and found that the $\mathrm{O}-\mathrm{C}$ residuals are fitted by a sinusoid with a period of four years and an amplitude of $1.2 \mathrm{~min}$. They also derived the geometry of the binary system using eclipse phases of the primary component and a bright spot. They found a mass ratio $q=0.72$ and an inclination of $i=85$ degrees. Baptista \& Catalan (2001) used high-speed photometry with eclipse mapping techniques to study the structure and time evolution of the accretion disc throughout the outburst cycle. Their analyses clearly proved the formation of a one-armed spiral structure on the rise to outburst. They also estimated the mass accretion rate at outburst maximum and in quiescence of about $\dot{M}_{\max }=10^{-8} M_{\odot} \mathrm{yr}^{-1}, \dot{M}_{\mathrm{q}}=10^{-9.1} M_{\odot} \mathrm{yr}^{-1}$, respectively. Shafter \& Holland (2003) analysed the eclipse profile of multicolour light curves with a parameter-fitting model. They derived a mass ratio $q<0.81$ and an inclination of $i>83 \mathrm{deg}$. Their revised ephemeris showed a cyclical variation with a period of five years. Knigge (2006) included EX Dra in his comprehensive study of spectral types of donor stars in CVs. Recently, Halevin $\&$ Henden (2008) analysed the dependence of the outburst cycle length from the other system parameters. They used a wavelet analysis to search for possible evolution of outburst cycles. Their wavelet map shows a rapid switching of the outburst time-scale from the 20-25 day cycle length before JD 52650 to one with an outburst time-scale of about 10-15 days. The main goal of this study is to publish the current period analyses based on all published data and our newly obtained photometry of this interesting object.

\section{Observations}

The eclipsing dwarf nova EX Dra was monitored from 2004 to 2011 at two observatories:

- Ondřejov Observatory, Czech Republic: a 0.65-m $(f / 3.6)$ reflecting telescope with the CCD cameras SBIG ST-8, Apogee AP7p or Moravian Instruments G2-3200 and VRI photometric filters during the period 2004-2011;

- Kolonica Saddle Observatory, Vihorlat, Slovak Republic: three different telescopes (1-m, $0.28-\mathrm{m}$ and $0.265-\mathrm{m})$ and three CCD cameras (Meade DSI Pro, SBIG ST-8, FLI PL1001E) were used with photometric $V R$ filters during 2008-2009. 


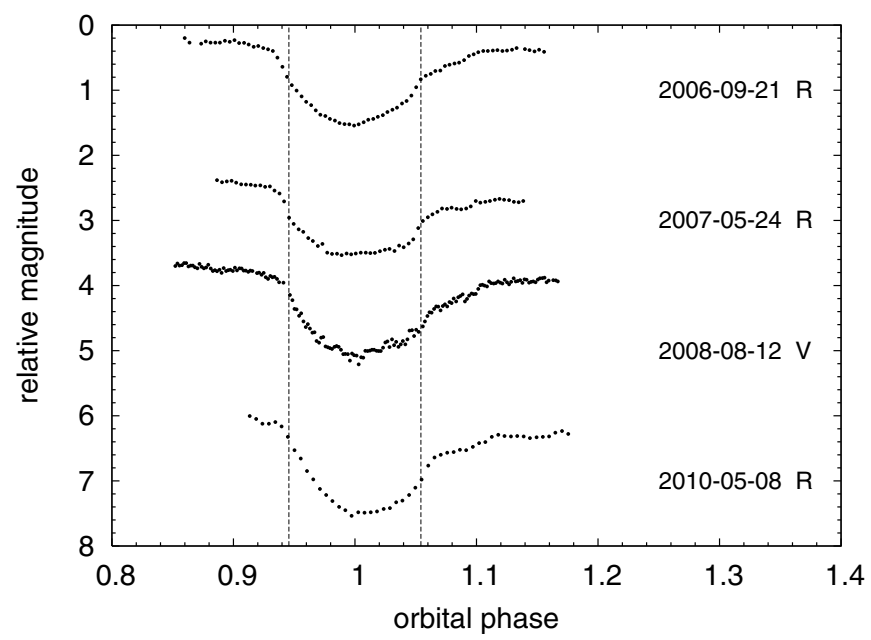

Fig. 1. Sample of eclipse light curves of EX Dra obtained at quiescence. The date of observation and the filter used are indicated. The orbital phase was calculated from the sinusoidal ephemeris given in Table 1. Two dashed lines denote the mid-ingress and the mid-egress of the white dwarf. For clarity, the individual light curves are shifted by 2 mag.

The CCD observations were reduced in a standard way. Арнот, a synthetic aperture photometry and astrometry software developed by Velen and Pravec at the Ondřejov observatory, and C-MunIPACK ${ }^{1}$ were routinely used for reduction of these CCD images. The data were dark-subtracted and flat-fielded and heliocentric correction was applied. In Table A.1 we summarise our CCD observations collected at Ondřejov and Kolonica observatories. At the Ondřejov we performed relative photometry with respect to the nearby comparison star GSC 4429-1414 $(V=14.4 \mathrm{mag})$. At Kolonica, the comparison star GSC 44291551 ( $V=14.61 \mathrm{mag}$ and $B-V=0.63$ ) was used. Timeseries were constructed by computing the magnitude difference between the variable and a comparison star. The sample of individual light curves of EX Dra observed during the quiescence are shown in Fig. 1, those obtained in the maximum are given in Fig. 2.

\section{Data analysis}

The mid-eclipse times were estimated from the mid-egress times of the white dwarf (WD). The bright spot (BS) ingress and egress depends on the variable's disc radius, therefore these times are not stable. On the other hand, the times of the WD ingress and egress are stable features. Mid-egresses of the WD and BS are better distinguishable than mid-ingresses, so we estimated the time mid-egress of the WD. Following Fiedler et al. (1997), we estimated the mid-egress time by fitting an approximating spline function to each light curve and by calculating its derivative. The time of mid-egress is the same as the time of local maximum. The procedure of estimating times of the WD egress is illustrated in Fig. 3. The uncertainty in determining mid-egress times $\left(\simeq 2.5 \times 10^{-4} \mathrm{~d}\right)$ depends on the time resolution and signalto-noise ratio. Individual errors of minima times are given in Table A. 2 in parenthesis. The mid-eclipse timing is independent of the passband.

We determined new times of mid-egress only during the quiescence of EX Dra. The mid-egress of the WD at the maximum of the outburst is not clearly visible in the derivate curve.

\footnotetext{
1 http://c-munipack. sourceforge.net/
}

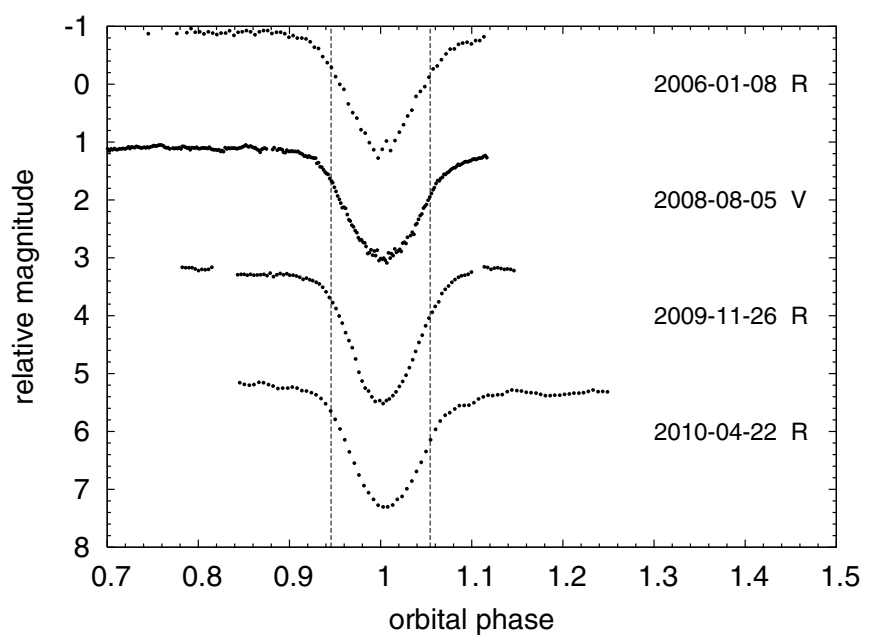

Fig. 2. Same as Fig. 1 but for the light curves obtained at maximum. The eclipses are deeper and more symmetric.

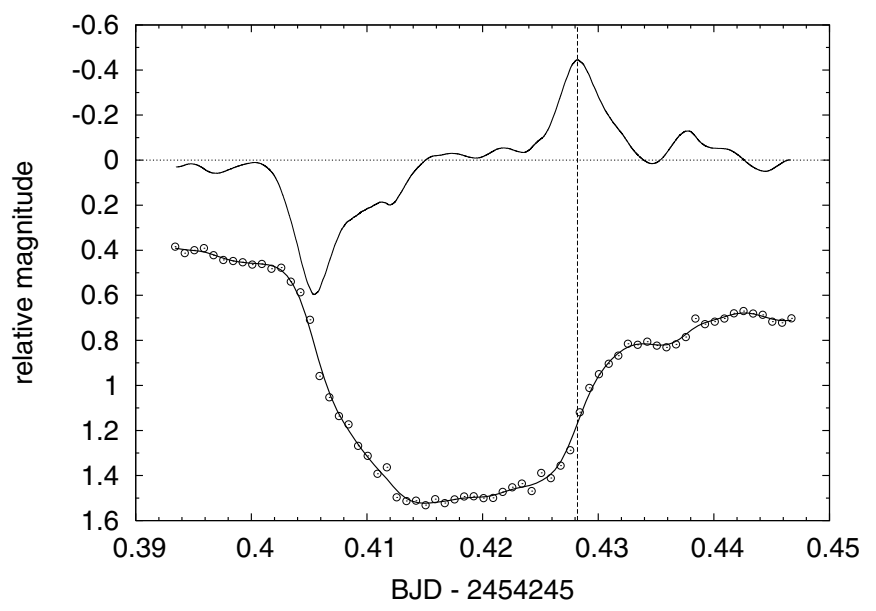

Fig. 3. Light curve of EX Dra in $R$ filter obtained on 24 May 2007. Individual measurements are denoted by circles, an approximate cubic spline function fitted to these points is indicated by the solid curve. For clarity, the derivative curve in arbitrary units is also plotted in the upper part of the diagram. The vertical line represents the time of the white dwarf egress.

Moreover, in 2004 EX Dra switched its state (Halevin \& Henden 2008) and the frequency of outbursts became higher, therefore it is less often in quiescence than before 2004. Thus, the light curves measured in more than half the nights are useless for estimating the mid-egress times.

Following Wood et al. (1985), we also calculated the eclipse width of the WD. We obtained $\Delta t_{\text {eclipse }}=0.0228$ day, which is very close to the value obtained by Baptista et al. (2000) and Shafter \& Holland (2003). We applied this value to all times of the mid-eclipse.

The difference between the universal time (UT) and the barycentric dynamical time (TDB) has changed by $9 \mathrm{~s}$ over the 20 years of observations. Mid-eclipse timing was calculated on the barycentric Julian date in the barycentric dynamical time $\mathrm{BJD}_{\mathrm{TDB}}$, according to Eastman et al. (2010). All these new times are collected in Table A.2.

\section{Period analysis}

Many eclipse timings for EX Dra suitable for the period analyses have been reported in the literature. Besides new minima given 
Table 1. Ephemerides of EX Dra.

\begin{tabular}{lc}
\hline \hline Quadratic ephemeris: & \\
Min. $=$ BJD $T_{0}+P_{0} \cdot E+Q \cdot E^{2}$ \\
\\
$T_{0}=2452474.8040(1) \mathrm{d}$ & $P_{0}=0.209937399(13) \mathrm{d}$ \\
$Q=1.02(7) \times 10^{-11} \mathrm{~d}$ & \\
$\chi_{\text {quad }}^{2}=1936$ & $v_{\text {quad }}=94$ \\
\hline Sinusoidal ephemeris: & \\
Min. $=$ BJD $T_{0}+P_{0} \cdot E+A \cdot \sin 2 \pi\left(\frac{E-B}{D}\right)$ \\
\\
$T_{0}=2452474.80513(10) \mathrm{d}$ & $P_{0}=0.209937316(13) \mathrm{d}$ \\
$A=0.00184(9) \mathrm{d}$ & $B=3520(360)$ epochs \\
$D=36600(2300)$ epochs $=7700(500) \mathrm{d}=21.1(1.3) \mathrm{yr}$ \\
$\chi_{\sin }^{2}=929$ & $v_{\sin }=91$ \\
\hline
\end{tabular}

in Table A.2, we also added the numerous timings obtained by Fiedler et al. (1997), Baptista et al. (2000) and Shafter \& Holland (2003). A total of 97 reliable times of minimum light were used in our dataset. Including all mid-eclipse times found in the literature and our new data set, we suggested as a first attempt that EX Dra exhibits a continuous period increase described by the quadratic elements. In accordance with previous authors we also suggested that the cyclical period change is represented by the sinusoidal ephemeris. The solutions of the least-squares fitting are collected in Table 1. The corresponding uncertainties are asymptotic standard deviations and are given in parenthesis. The values of $\chi^{2}$ and degrees of freedom $v$ of both ephemeris are also included. The data points were weighted by the inverse of the squares of the uncertainties in the mid-eclipse times. The corresponding $\mathrm{O}-\mathrm{C}$ diagrams are plotted in Fig. 4.

A sinusoidal ephemeris with a period of about 21 years fits the data better than the quadratic ephemeris. The $\chi_{\text {quad }}^{2}$ is more than twice greater than $\chi_{\text {sin }}^{2}$ (see Table 1). The current trend is a decreasing orbital period and the last mid-eclipse times deviate more from the quadratic ephemeris (see the upper panel of Fig. 4). Therefore, the sinusoidal ephemeris is more significant than the quadratic one. On the other hand, the eclipse timings show substantial deviations from the best sinusoidal fit, the $\chi_{\sin }^{2}=929 \gg 1$ indicates that this ephemeris is not a complete description of the period changes.

\section{Discussion}

The best current explanation for the observed cyclical period modulation is a solar-type magnetic activity in the secondary star, which was proposed to explain these modulations by Applegate (1992). Variations in the internal magnetic torque cause a cyclical exchange of the internal angular momentum between an inner and an outer convective shell. This initiates cyclical changes in the oblateness and quadrupole moment. As a consequence of the change of the secondary Roche lobe, the mass-transfer also changes (Richman et al. 1994). When the orbital period is shortest, the secondary's outer layer rotates faster, the secondary is most oblate and vice versa. The fractional period change $\Delta P / P$ is related to the amplitude $\Delta(\mathrm{O}-\mathrm{C})$ and to the cycle length $P_{\text {mod }}$ of the modulation (Applegate 1992):

$\frac{\Delta P}{P}=2 \pi \frac{\Delta(\mathrm{O}-\mathrm{C})}{P_{\bmod }}=4 \pi \frac{A}{D}$.

Using the values of $A$ and $D$ given above we find $\Delta P / P=$ $3.01 \times 10^{-6}$, i.e. $\log \Delta P / P=-5.52$. All CVs with observed period modulations and corresponding fractional period changes
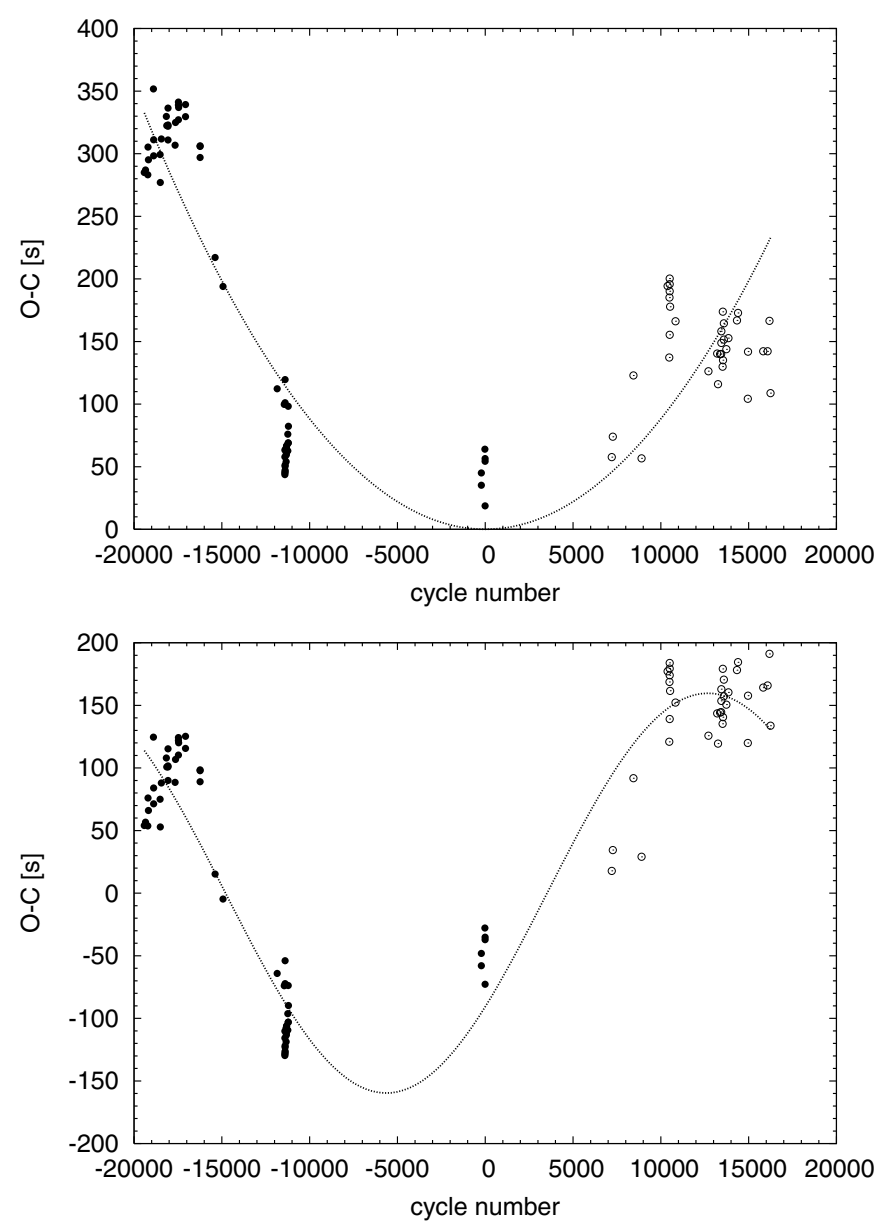

Fig. 4. Current O-C diagram for EX Dra. New eclipse times are denoted by circles, those obtained from the literature are depicted by dots. Top: $\mathrm{O}-\mathrm{C}$ diagram with respect to the linear term of quadratic ephemeris. The curve represents the best-fit quadratic ephemeris. Bottom: O-C diagram with respect to the linear term of sinusoidal ephemeris. The curve denotes the best-fit sinusoidal ephemeris with a period of about 21 years.

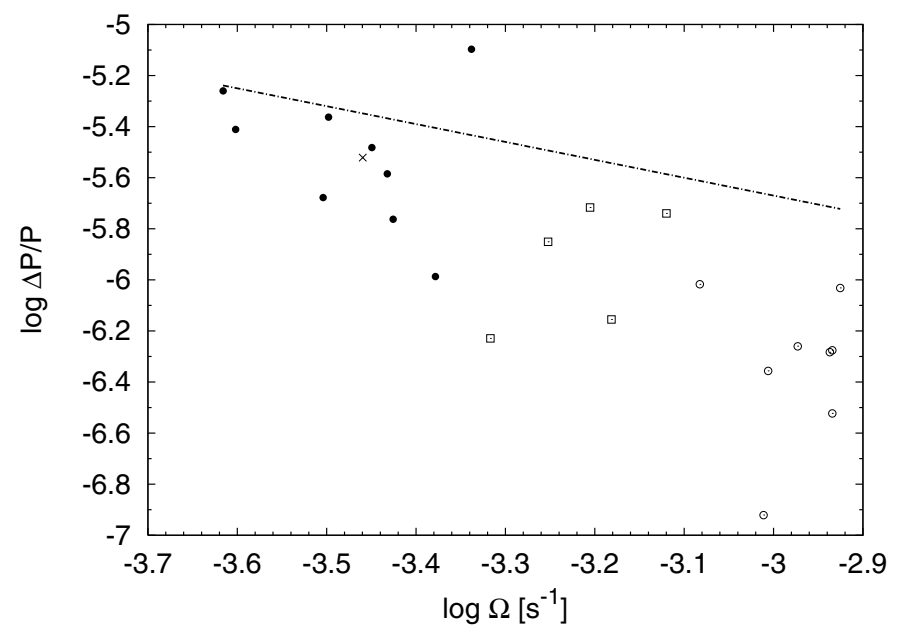

Fig. 5. Diagram of the fractional period change $\Delta P / P$ versus the angular velocity $\Omega$ of the active component star for CVs according to Table 2 . The position of EX Dra is indicated by a cross, the other long-period $\mathrm{CVs}$, short-period CVs and pre-CVs in the period gap are denoted by dots, circles, and open squares, respectively. The best linear fit to data above the period gap derived by Borges et al. (2008) $\Delta P / P \propto \Omega^{-0.7}$ is indicated by the dot-dashed line. 
Table 2. Observed period modulation in CVs.

\begin{tabular}{lcccc}
\hline \hline Object & $\begin{array}{c}P_{\text {orb }} \\
{[\mathrm{h}]}\end{array}$ & $\begin{array}{c}P_{\text {mod }} \\
{[\mathrm{yr}]}\end{array}$ & $\begin{array}{c}\Delta P / P \\
\times 10^{-6}\end{array}$ & Ref. \\
\hline V4140 Sgr & 1.47 & 6.9 & 0.93 & 1 \\
DP Leo & 1.50 & 23.8 & 0.53 & 2 \\
V2051 Oph & 1.50 & 22 & 0.30 & 1 \\
OY Car & 1.51 & 35 & 0.52 & 3 \\
EX Hya & 1.64 & 17.5 & 0.55 & 4 \\
HT Cas & 1.77 & 36 & 0.44 & 5 \\
Z Cha & 1.79 & 32.6 & 0.12 & 6 \\
UZ For & 2.11 & 23.4 & 0.96 & 7 \\
HS 0705+6700 & 2.30 & 15.7 & 1.82 & 8 \\
HS 2231+2441 & 2.65 & 15.7 & 0.70 & 8 \\
HW Vir & 2.80 & 15.7 & 1.92 & 9 \\
NN Ser & 3.12 & 7.56 & 1.41 & 10 \\
QS Vir & 3.62 & 7.86 & 0.59 & 11 \\
IP Peg & 3.80 & 4.7 & 8.00 & 12 \\
U Gem & 4.17 & 17.4 & 1.03 & 13 \\
DQ Her & 4.65 & 17.7 & 1.73 & 14 \\
UX UMa & 4.72 & $7.1,10.7,30.4$ & 2.60 & 15 \\
T Aur & 4.91 & 24 & 3.30 & 16 \\
EX Dra & 5.03 & 21 & 3.01 & this work \\
TV Col & 5.49 & 31 & 4.33 & 17 \\
RW Tri & 5.57 & $7.6,13.6$ & 2.10 & 18 \\
EM Cyg & 6.98 & 17.74 & 3.88 & 19 \\
AC Cnc & 7.21 & 16.2 & 5.50 & 20 \\
\hline
\end{tabular}

References. (1) Baptista et al. (2003); (2) Qian et al. (2010a); (3) Greenhill et al. (2006); (4) Helier \& Sproats (1992); (5) Borges et al. (2008); (6) Dai et al. (2009); (7) Dai et al. (2010b); (8) Qian et al. (2010c); (9) Qian et al. (2008); (10) Qian et al. (2009); (11) Qian et al. (2010b); (12) Wolf et al. (1993); (13) Dai \& Qian (2009a); (14) Dai \& Qian (2009b); (15) Rubenstein et al. (1991); (16) Dai \& Qian (2010a); (17) Dai et al. (2010a); (18) Robinson et al. (1991); (19) Dai \& Qian (2010b); (20) Qian et al. (2007).

are listed in Table 2. The diagram of the period change versus the angular velocity is plotted in Fig. 5.

The short-period CVs also show period cyclical changes. The secondaries of the short-period CVs are fully convective, so if the period change is caused by magnetic activity, it would mean that the fully convective dwarfs show magnetic cycles. Borges et al. (2008) compared CVs below and above the period gap. They calculated a relation $\triangle P / P \propto \Omega^{-0.7}$ for the long-period CVs, RS CVn, W UMa and Algols and showed that the shortperiod CVs significantly deviate from this relation. This is also visible in Fig. 5. Recently observed pre-CVs with period modulation in the period gap are also plotted.

\section{Conclusions}

We completed a new period study of EX Dra by performing an $\mathrm{O}-\mathrm{C}$ diagram analysis and adopting a complete list of published and newly observed times of mid-eclipses. New eclipse timings were measured from the light curves in quiescence, and revised elements were obtained. The residuals with respect to the linear ephemeris show a cyclical behaviour. We cannot confirm the short four-year nor the five-year cyclical period of O-C values announced by Baptista et al. (2000) and Shafter \& Holland (2003). The current O-C data present a $21 \mathrm{yr}$ period modulation with a semi-amplitude of $2.5 \mathrm{~min}$. Nevertheless, eclipse timings show significant deviations from the best sinusoidal fit, which indicates that this ephemeris is not the only and complete description of the data. It is highly desirable to continue with the high-precision photometric monitoring to obtain precise eclipse timings of this interesting system. Acknowledgements. This investigation was supported by the Research Program
MSM0021620860 Physical Study of objects and processes in the Solar System
and in Astrophysics of the Ministry of Education of the Czech Republic and par-
tially by the Czech Science Foundation, grants $205 / 04 / 2063,205 / 06 / 0217$, and
in its final stage by the grant P205/10/0715. The research of PAD was supported
by the APVV grants LPP-0049-06 and LPP-0024-09 The authors thank former
students: M. Bílek, J. Čechura, I. Ebrová, S. Koberle, S. Poddaný, V. Sidorin,
J. Verfl, and Drs. J. Ďurech and P. Zasche, for their assistance during the pho-
tometric observations. The following internet-based resources were used in re-
search for this paper: the SIMBAD database and the VizieR service operated at
CDS, Strasbourg, France; the NASA's Astrophysics Data System Bibliographic
Services; and the AAVSO database.

\section{References}

Applegate, J. H. 1992, ApJ, 385, 621

Bade, N., Hagen, H.-J., \& Reimers, D. 1989, in 23rd ESLAB Symp., ESA SP296,883

Baptista, R., \& Catalán, M. S. 2001, MNRAS, 324, 599

Baptista, R., Catalán, M. S., \& Costa, L. 2000, MNRAS, 316, 526

Baptista, R., Borges B. W., Bond, H. E., et al. 2003, MNRAS, 345, 889

Barwig, H., Fiedler, H., Reimers, D., \& Bade, N. 1993, in Compact Stars in Binary Systems, IAU Symp., 165, 89

Borges, B. W., Baptista, R., Papadimitriou, C., \& Giannakis, O. 2008, A\&A, 480,481

Dai, Z. B., \& Qian, S. B. 2009a, Ap\&SS, 321, 91

Dai, Z. B., \& Qian, S. B. 2009b, A\&A, 503, 883

Dai, Z. B., \& Qian, S. B. 2010a, New Astron., 15, 380

Dai, Z. B., \& Qian, S. B. 2010b, PASJ, 62, 965

Dai, Z. B., Qian, S. B., \& Fernándes Lajús, E. 2009, ApJ, 703, 109

Dai, Z. B., Qian, S. B., Fernándes Lajús, E., et al. 2010a, Ap\&SS, 330, 243

Dai, Z. B., Qian, S. B., Fernándes Lajús, E., et al. 2010b, MNRAS, 409, 1195

Eastman, J., Siverd, R., \& Gaudi, B. S. 2010, PASP, 122, 935

Fiedler, H., Barwig, H., \& Mantel, K. H. 1997, A\&A, 327, 173

Greenhill, J. G., Hill, K. M., Dieters, S., et al. 2006, MNRAS, 372, 1129

Halevin, A. V., \& Henden, A. A. 2008, IBVS, 5833

Helier, C., \& Sproats, L. N. 1992, IBVS, 3724, 1

Knigge, C. 2006, MNRAS, 373, 484

Lanza, A. F., Rodono, M., \& Rosner, R. 1998, MNRAS, 296, 893

Qian, S. B., Dai, Z. B., He, J. J., et al. 2007, A\&A, 466, 589

Qian, S. B., Dai, Z. B., Zhu, L. Y., et al. 2008, ApJ, 689, 49

Qian, S. B., Dai, Z. B., Liao, W. P., et al. 2009, ApJ, 706, 96

Qian, S. B., Liao, W. P., Zhu, L. Y., et al. 2010a, ApJ, 708, 66

Qian, S. B., Liao, W. P., Zhu, L. Y., et al. 2010b, MNRAS, 401, L34

Qian, S. B., Zhu, L. Y., Liu, L., et al. 2010c, Ap\&SS, 329, 113

Richman, H. R., Applegate, J. H., \& Patterson, J. 1994, PASP, 106, 1075

Robinson, E. L., Shetrone, M. D., \& Africano, L. J. 1991, AJ, 102, 1176

Rubenstein, E. P., Patterson, J., \& Africano, J. L. 1991, PASP, 103, 1258

Shafter, A., \& Holland, J. 2003, PASP, 115, 1105

Wolf, S., Mantel, K. H., Horne, K., et al. 1993, A\&A, 273, 160

Wood, J. H., Irwin, M. J., \& Pringle, J. E. 1985, MNRAS, 214, 475 


\section{Appendix A: Tables}

Table A.1. Journal of the observations of EX Dra.

\begin{tabular}{|c|c|c|c|c|c|}
\hline Date & $\begin{array}{l}\text { Start } \\
{[\mathrm{UT}]}\end{array}$ & $\begin{array}{c}\text { Exp. } \\
\text { Time [s] }\end{array}$ & $\begin{array}{l}\text { No. of } \\
\text { exp. }\end{array}$ & Band & $\begin{array}{c}\text { Observatory } \\
\text { remarks }\end{array}$ \\
\hline 2004 Sep. 02 & $19: 20$ & 60 & 43 & $R$ & $\mathrm{O}$ \\
\hline 2004 Sep. 07 & $21: 10$ & 60 & 31 & $R$ & $\mathrm{O}$ \\
\hline 2005 May 26 & $20: 07$ & 60 & 38 & $R$ & $\mathrm{O}$ \\
\hline 2006 Jan. 09 & $03: 53$ & 80 & 73 & $R$ & $\mathrm{O}$ \\
\hline 2006 Jul. 17 & $23: 26$ & 60 & 53 & $R$ & $\mathrm{O}$ \\
\hline 2006 Sep. 09 & $22: 20$ & 60 & 47 & $R$ & $\mathrm{O}, \mathrm{M}$ \\
\hline 2006 Sep. 21 & $21: 22$ & 60 & 74 & $R$ & $\mathrm{O}, \mathrm{M}$ \\
\hline 2006 Oct. 12 & $21: 29$ & 60 & 57 & $R$ & $\mathrm{O}$ \\
\hline 2007 Apr. 19 & $20: 10$ & 60 & 58 & $R$ & $\mathrm{O}$ \\
\hline 2007 May 11 & $00: 11$ & 60 & 71 & $R$ & $\mathrm{O}$ \\
\hline 2007 May 24 & $21: 26$ & 60 & 65 & $R$ & $\mathrm{O}, \mathrm{M}$ \\
\hline 2007 Aug. 22 & $23: 27$ & 60 & 61 & $R$ & $\mathrm{O}$ \\
\hline 2007 Aug. 30 & $22: 15$ & 60 & 60 & $R$ & $\mathrm{O}, \mathrm{M}$ \\
\hline 2008 Jun. 30 & $20: 35$ & 30 & 185 & $V$ & K \\
\hline 2008 Jul. 01 & $20: 30$ & 30 & 503 & $V$ & $\mathrm{~K}$ \\
\hline 2008 Jul. 02 & $20: 16$ & 30 & 182 & $V$ & $\mathrm{~K}$ \\
\hline 2008 Jul. 06 & $20: 20$ & 30 & 108 & $V$ & $\mathrm{~K}, \mathrm{M}$ \\
\hline 2008 Jul. 11 & $20: 33$ & 30 & 210 & $V$ & K \\
\hline 2008 Jul. 12 & $21: 01$ & 30 & 346 & $V$ & $\mathrm{~K}$ \\
\hline 2008 Jul. 27 & $19: 44$ & 30 & 166 & $V$ & $\mathrm{~K}, \mathrm{M}$ \\
\hline 2008 Jul. 28 & $19: 46$ & 30 & 147 & $V$ & $\mathrm{~K}, \mathrm{M}$ \\
\hline 2008 Jul. 29 & $22: 51$ & 30 & 245 & $V$ & $\mathrm{~K}, \mathrm{M}$ \\
\hline 2008 Jul. 30 & $23: 37$ & 60 & 93 & $R$ & $\mathrm{~K}, \mathrm{M}$ \\
\hline 2008 Jul. 31 & $19: 59$ & 30 & 238 & $V$ & $\mathrm{~K}, \mathrm{M}$ \\
\hline 2008 Aug. 01 & $20: 24$ & 30 & 501 & $V$ & $\mathrm{~K}, \mathrm{M}$ \\
\hline 2008 Aug. 05 & $19: 46$ & 30 & 258 & $V$ & K \\
\hline 2008 Aug. 07 & $23: 28$ & 60 & 84 & $R$ & $\mathrm{~K}, \mathrm{M}$ \\
\hline 2008 Aug. 12 & 19:02 & 30 & 168 & $V$ & K \\
\hline 2008 Aug. 31 & $18: 43$ & 30 & 541 & $V$ & $\mathrm{~K}$ \\
\hline 2008 Sep. 02 & $18: 17$ & 30 & 715 & $V$ & $\mathrm{~K}$ \\
\hline 2008 Sep. 03 & $18: 46$ & 30 & 550 & $V$ & $\mathrm{~K}$ \\
\hline 2008 Oct. 09 & $17: 24$ & 30 & 309 & $V$ & $\mathrm{~K}, \mathrm{M}$ \\
\hline 2009 Sep. 12 & 18:08 & 30 & 90 & $R$ & $\mathrm{~K}$ \\
\hline 2009 Nov. 05 & $16: 34$ & 60 & 68 & $R$ & $\mathrm{O}, \mathrm{M}$ \\
\hline 2009 Nov. 26 & $16: 00$ & 60 & 92 & $R$ & $\mathrm{O}$ \\
\hline 2010 Feb. 18 & $21: 25$ & 60 & 72 & $R$ & $\mathrm{O}, \mathrm{M}$ \\
\hline 2010 Feb. 22 & $19: 53$ & 90 & 104 & $R$ & $\mathrm{O}$ \\
\hline 2010 Mar. 01 & $23: 27$ & 60 & 61 & $R$ & $\mathrm{O}, \mathrm{M}$ \\
\hline 2010 Mar. 25 & $22: 08$ & 90 & 144 & $V$ & $\mathrm{O}, \mathrm{M}$ \\
\hline 2010 Apr. 07 & $22: 10$ & 60 & 210 & $R$ & $\mathrm{O}, \mathrm{M}$ \\
\hline 2010 Apr. 08 & $18: 38$ & 90 & 235 & $V$ & $\mathrm{O}, 2 \mathrm{M}$ \\
\hline 2010 Apr. 22 & $20: 19$ & 90 & 250 & $R$ & $\mathrm{O}$ \\
\hline 2010 Apr. 25 & $23: 54$ & 90 & 114 & $R$ & $\mathrm{O}, \mathrm{M}$ \\
\hline 2010 Apr. 28 & $23: 54$ & 90 & 150 & $R$ & $\mathrm{O}, \mathrm{M}$ \\
\hline 2010 Apr. 29 & $21: 10$ & 90 & 210 & $R$ & $\mathrm{O}, \mathrm{M}$ \\
\hline 2010 May 08 & $19: 35$ & 90 & 145 & $R$ & $\mathrm{O}, 2 \mathrm{M}$ \\
\hline 2010 May 27 & $21: 28$ & 90 & 69 & $R$ & $\mathrm{O}$ \\
\hline 2010 Jun. 10 & $23: 30$ & 90 & 48 & $V$ & $\mathrm{O}, \mathrm{M}$ \\
\hline 2010 Jun. 21 & $20: 48$ & 90 & 59 & $R$ & $\mathrm{O}$ \\
\hline 2010 Jun. 24 & $22: 43$ & 90 & 87 & $R$ & $\mathrm{O}$ \\
\hline 2010 Jun. 30 & $22: 55$ & 90 & 50 & $R$ & $\mathrm{O}$ \\
\hline 2010 Jul. 03 & $20: 28$ & 90 & 66 & $R$ & $\mathrm{O}, \mathrm{M}$ \\
\hline 2010 Aug. 22 & $20: 23$ & 90 & 19 & $R$ & $\mathrm{O}$ \\
\hline 2010 Sep. 30 & $18: 18$ & 90 & 54 & $R$ & $\mathrm{O}$ \\
\hline 2010 Oct. 14 & 17.03 & 60 & 93 & $R$ & $\mathrm{O}, \mathrm{M}$ \\
\hline 2010 Oct. 21 & $17: 02$ & 60 & 259 & $I$ & $\mathrm{O}$ \\
\hline 2010 Oct. 24 & $18: 00$ & 60 & 103 & $I$ & $\mathrm{O}$ \\
\hline 2010 Oct. 26 & $16: 48$ & 60 & 147 & $I$ & $\mathrm{O}, \mathrm{M}$ \\
\hline 2010 Oct. 30 & $17: 57$ & 60 & 191 & $I$ & $\mathrm{O}$ \\
\hline 2010 Oct. 31 & $18: 45$ & 60 & 175 & $I$ & $\mathrm{O}$ \\
\hline 2010 Nov. 11 & $16: 15$ & 60 & 52 & $R$ & $\mathrm{O}$ \\
\hline 2011 Jan. 06 & $00: 56$ & 90 & 106 & $R$ & $\mathrm{O}$ \\
\hline 2011 Feb. 22 & 02:08 & 90 & 75 & $R$ & $\mathrm{O}, \mathrm{M}$ \\
\hline 2011 Feb. 25 & 01:07 & 60 & 63 & $R$ & $\mathrm{O}, \mathrm{M}$ \\
\hline 2011 Apr. 17 & $21: 46$ & 60 & 46 & $R$ & $\mathrm{O}$ \\
\hline 2011 Apr. 21 & $21: 05$ & 60 & 63 & $R$ & $\mathrm{O}$ \\
\hline 2011 Aug. 03 & $19: 39$ & 60 & 33 & $R$ & $\mathrm{O}$ \\
\hline 2011 Aug. 10 & $22: 43$ & 60 & 59 & $R$ & $\mathrm{O}$ \\
\hline 2011 Aug. 25 & $20: 36$ & 60 & 49 & $R$ & $\mathrm{O}, \mathrm{M}$ \\
\hline
\end{tabular}

Table A.1. continued.

\begin{tabular}{lccccc}
\hline \hline Date & $\begin{array}{c}\text { Start } \\
\text { [UT] }\end{array}$ & $\begin{array}{c}\text { Exp. } \\
\text { Time [s] }\end{array}$ & $\begin{array}{c}\text { No. of } \\
\text { exp. }\end{array}$ & Band & $\begin{array}{c}\text { Observatory } \\
\text { remarks }\end{array}$ \\
\hline 2011 Aug. 26 & $21: 39$ & 60 & 45 & $R$ & $\mathrm{O}$ \\
2011 Sep. 15 & $20: 27$ & 60 & 44 & $R$ & $\mathrm{O}$ \\
2011 Oct. 04 & $17: 52$ & 60 & 47 & clear & $\mathrm{O}$ \\
2011 Oct. 09 & $19: 10$ & 30 & 53 & clear & $\mathrm{O}$ \\
2011 Oct. 14 & $19: 43$ & 30 & 88 & clear & O, M \\
2011 Nov. 06 & $16: 52$ & 30 & 184 & clear & O, M \\
2011 Nov. 19 & $17: 15$ & 40 & 127 & clear & O, M \\
\hline
\end{tabular}

Notes. Observatory: K ... Kolonica, O ... Ondřejov. Remarks: M ... used for timings.

Table A.2. Times of minimum light of EX Dra obtained in quiescence.

\begin{tabular}{|c|c|c|}
\hline $\begin{array}{l}\text { BJD- } \\
2400000\end{array}$ & Epoch & Filter \\
\hline $53988.4534(3)$ & 7210 & $R$ \\
\hline $54000.4200(3)$ & 7267 & $R$ \\
\hline $54245.4175(3)$ & 8434 & $R$ \\
\hline $54343.4575(3)$ & 8901 & $R$ \\
\hline $54654.3764(3)$ & 10382 & V \\
\hline $54675.3695(3)$ & 10482 & V \\
\hline $54676.4197(3)$ & 10487 & V \\
\hline $54677.4695(3)$ & 10492 & V \\
\hline $54678.5193(3)$ & 10497 & $R$ \\
\hline $54679.3585(3)$ & 10501 & V \\
\hline $54680.4086(3)$ & 10506 & $V$ \\
\hline $54686.4966(3)$ & 10535 & $R$ \\
\hline $54749.2678(3)$ & 10834 & V \\
\hline $55141.2204(3)$ & 12701 & $R$ \\
\hline $55246.3992(3)$ & 13202 & $R$ \\
\hline $55257.5256(3)$ & 13255 & $R$ \\
\hline $55281.4588(3)$ & 13369 & V \\
\hline $55294.4749(3)$ & 13431 & $R$ \\
\hline $55295.3149(3)$ & 13435 & V \\
\hline $55295.5247(3)$ & 13436 & $V$ \\
\hline $55312.5294(3)$ & 13517 & $R$ \\
\hline $55315.4690(3)$ & 13531 & $R$ \\
\hline $55316.5183(3)$ & 13536 & $R$ \\
\hline $55325.3358(3)$ & 13578 & $R$ \\
\hline $55325.5459(3)$ & 13579 & $R$ \\
\hline $55358.5058(3)$ & 13736 & $V$ \\
\hline $55381.3891(3)$ & 13845 & $R$ \\
\hline $55484.2586(3)$ & 14335 & $R$ \\
\hline $55496.2251(3)$ & 14392 & $I$ \\
\hline $55614.6290(3)$ & 14956 & $R$ \\
\hline $55617.5686(3)$ & 14970 & $R$ \\
\hline $55799.3744(3)$ & 15836 & $R$ \\
\hline $55849.3395(2)$ & 16074 & clear \\
\hline $55872.2229(2)$ & 16183 & clear \\
\hline $55885.2384(2)$ & 16245 & clear \\
\hline
\end{tabular}

